\title{
Image processing for a formation flying synthetic aperture telescope
}

Norihide Miyamura, Ryo Suzumoto, Satoshi Ikari, Shinichi Nakasuka

Norihide Miyamura, Ryo Suzumoto, Satoshi Ikari, Shinichi Nakasuka, "Image processing for a formation flying synthetic aperture telescope," Proc. SPIE 11852, International Conference on Space Optics - ICSO 2020, 118524W (11 June 2021); doi: 10.1117/12.2599818

SPIE Event: International Conference on Space Optics - ICSO 2021, 2021, Online Only 


\section{International Conference on Space Optics-ICSO 2020}

Virtual Conference

30 March-2 April 2021

Edited by Bruno Cugny, Zoran Sodnik, and Nikos Karafolas
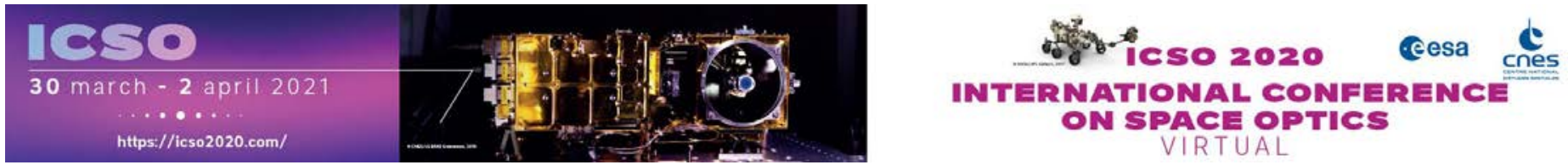

\section{Image processing for a formation flying synthetic aperture telescope}

\section{Cesa isoporecestings denes}




\title{
Image processing for a formation flying synthetic aperture telescope
}

\author{
Norihide Miyamura*a, Ryo Suzumoto ${ }^{\mathrm{b}}$, Satoshi Ikari ${ }^{\mathrm{b}}$, Shinichi Nakasuka ${ }^{\mathrm{b}}$ \\ ${ }^{a}$ Meisei University, 2-1-1 Hodokubo, Hino, Tokyo, JAPAN 191-8506; ${ }^{\circ}$ The University of Tokyo, 7- \\ 3-1 Hongo, Bunkyo, Tokyo, JAPAN 113-8656
}

\begin{abstract}
Small satellites have been used for remote sensing with a spatial resolution of several meters from LEO in recent years. However, it is difficult to increase temporal resolution for LEO remote sensing due to the short orbital period. Therefore, GEO remote sensing which enables observation of high temporal resolution from GEO or its nearby orbit is getting important. In order to obtain enough spatial resolution in GEO remote sensing, an optical system having a diameter of several meters is required because of the diffraction limit. It takes huge cost to realize such a large diameter primary mirror due to manufacturability and required accuracy. To address this problem, we propose a synthetic aperture telescope by small satellites formation flying. The synthetic aperture telescope is composed of several mirror satellites constituting a primary mirror of the telescope and an imaging satellite having a focal plane assembly. By optically synthesizing the light collected by each mirror satellite with the imaging satellite, a virtual large aperture telescope is constructed. In this paper, we assume the observation at near infrared to short wavelength infrared and show the specifications of the system. The optical performance of the synthetic aperture telescope and the image processing method used to extract high spatial frequency information from the observed images are also described.
\end{abstract}

Keywords: Formation flying, adaptive optics, active optics, wavefront sensing

\section{INTRODUCTION}

Spatial resolution of several meters from low earth orbit (LEO) has been realized in small satellite remote sensing in recent years. For example, the "Hodoyoshi-4" launched in 2014 achieved spatial resolution of $6 \mathrm{~m}$ from an altitude of $600 \mathrm{~km}$. However, it is difficult to increase temporal resolution in LEO remote sensing. For disaster monitoring, high temporal resolution of about 10 minutes is required. In order to realize that with small satellites on LEO, hundreds of satellites are necessary. Therefore, the geostationary orbit (GEO) remote sensing which enables observation of high temporal resolution from GEO, or its nearby orbit is getting important. On the other hand, because of the long distance between a satellite and a ground target, spatial resolution in GEO remote sensing decreases by 50 to 60 times compared to that in LEO remote sensing. In order to obtain enough spatial resolution in GEO remote sensing, an optical system having a diameter of several meters is required because of the diffraction limit. It takes huge cost to realize the optical system with such a large diameter due to manufacturability and required accuracy.

To address the problem, we propose Formation Flying Synthetic Aperture Telescope (FFSAT). A schematic view of the FFSAT is shown in Figure 1. The FFSAT is composed of mirror satellites constituting a primary mirror of the telescope and an imaging satellite having a focal plane assembly. By optically synthesizing the light collected by each mirror satellite with the imaging satellite, a virtual large aperture telescope is constructed. Here, the issue is whether the image can be synthesized by adjusting the optical system with an accuracy of $1 / 10$ of the observation wavelength. We aim to achieve the accuracy by combining small satellite formation flying of micrometer order with an adaptive optics system distributed in mirror satellites and the imaging satellite. The adaptive optics system compensates wavefront aberrations using multiple degree of freedom mirror mounts and deformable mirrors (DMs).

In this paper, from the viewpoint of disaster monitoring applications and required accuracy for the optical system, observations at near infrared to short wavelength infrared are assumed and required specifications are shown. Then, The optical performance of the synthetic aperture telescope and the image processing method used to extract high spatial frequency information from the observed images are also described.

*norihide.miyamura@meisei-u.ac.jp; phone 8142 591-5895 


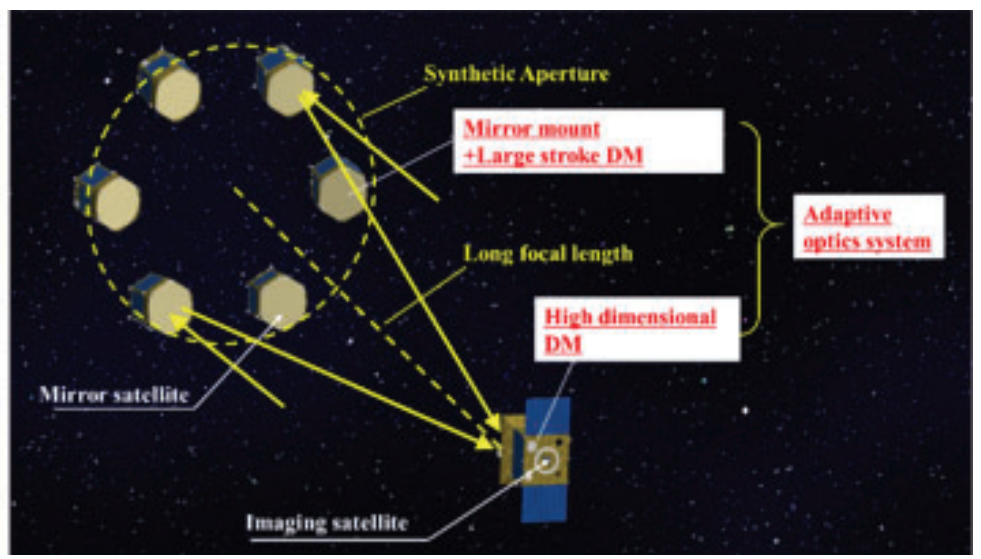

Figure. 1. The concept of the FFSAT. Six mirror satellites make up the primary mirror, and images are acquired by imaging satellites to construct a telescope with a long focal length and large aperture in orbit. The high precision stage of the mirror satellites and the adaptive optics of the imaging satellites will be used to image the light flux from each mirror satellite on the imaging satellite.

\section{CONCEPT OF THE SYNTHETIC APERTURE TELESCOPE SYSTEM}

The results of the ray tracing simulation of FFSAT, calculated according to the specifications in Table 1, are shown in Figure 2. The FFSAT consists of formation flying with six mirror satellites and one imaging satellite. Six mirror satellites will be placed in geostationary orbit and imaging satellites will be placed in inner orbit. Since the formation needs to be maintained including imaging satellites with different orbital radii, fuel-based orbit control is necessary. The large diameter and sparse primary mirror is formed by six mirror satellites. The input ray of each mirror satellite is gathered in the imaging satellite. The mirror satellites use high-precision piezo stages to control the position and attitude of the mirrors, and the imaging satellite uses adaptive optics system with a deformable mirror to correct the wavefront aberration. The errors in optical path difference and wavefront aberrations caused by misalignment, thermal distortion, and insufficient mechanical precision are corrected by the cooperation of these control systems, and images are acquired by the imaging satellite.

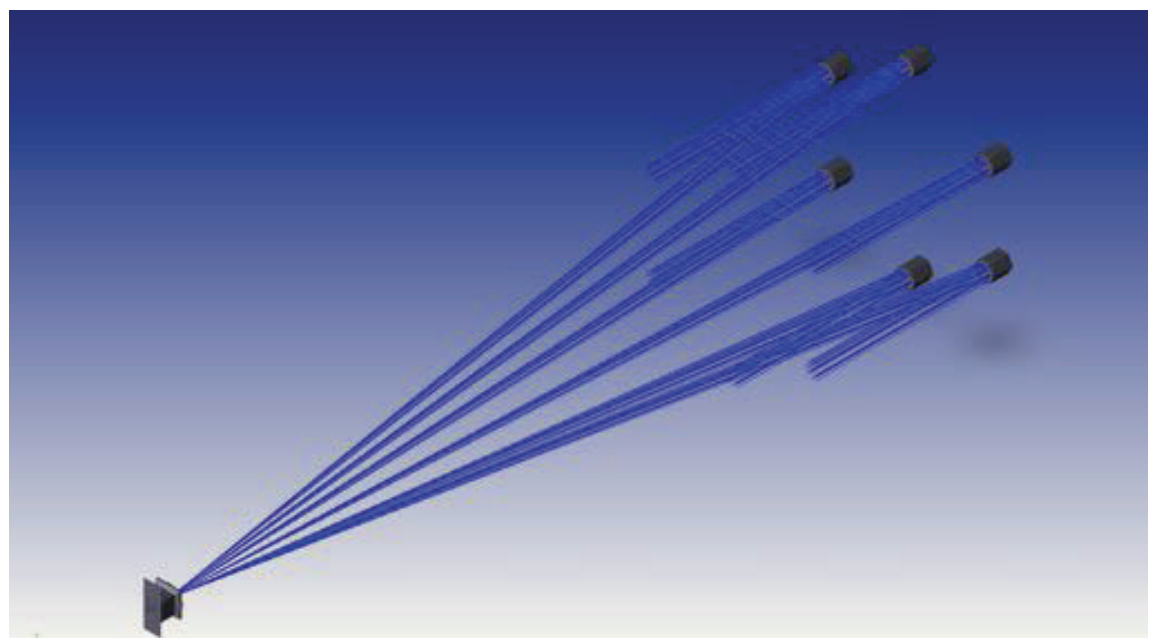

Figure. 2. Simulation results of ray tracing when optical parameters are set in the FFSAT mechanical model. The rays are calculated results when observing a point light source on the optical axis and at infinite distance. 
Table 1. Specifications of the FFSAT system.

\begin{tabular}{lc}
\hline Items & Values \\
\hline Focal length $[\mathrm{m}]$ & 21.5 \\
Synthetic aperture diameter $[\mathrm{m}]$ & 5.82 \\
Pixel size $[\mu \mathrm{m}]$ & 18 \\
Number of pixels & $3000 \times 3000$ \\
\hline
\end{tabular}

\section{FORMULIZATION OF THE SYNTHETIC APERTURE TELESCOPE SYSTEM}

The layout of the aperture pupil composed by the mirror satellite and the wavefront aberration affect a point spread function (PSF). In the case of the field of view (FOV) of the sensor is sufficiently narrow that the PSF is constant in the region, an observed image for the extended object $o(u, v)$ at wavelength $\lambda$ is expressed as equation (1).

$$
i(u, v)=\sum_{\lambda} o_{\lambda}(u, v) * s_{\lambda}(u, v)+n(u, v),
$$

where $*$ indicate convolution and $(u, v)$ are image plane coordinates.

The PSF of the FFSAT is calculated using a Fourier transform of a generalized pupil function $P_{\lambda}(x, y)$ as shown in equation (3), where $(x, y)$ are pupil plane coordinates. A generalized pupil function is calculated using the shape of the pupil $A(x, y)$ and a wavefront function $\Phi(x, y)$.

$$
\begin{gathered}
s_{\lambda}(u, v)=\left\{F\left[P_{\lambda}\left(\frac{u}{\lambda f}, \frac{v}{\lambda f}\right)\right]\right\}^{2}, \\
P_{\lambda}(x, y)=A(x, y) \exp \{i \Phi(x, y)\}, \\
A(x, y)=\sum_{i=1}^{N} A_{i}(x, y), \\
A_{i}(x, y)=\left\{\begin{array}{cc}
1, & \left(x-x_{c i}\right)^{2}+\left(y-y_{c i}\right)^{2} \leq r_{i}, \\
0, & \text { otherwise }
\end{array},\right.
\end{gathered}
$$

where $F[]$ denotes a Fourier transform. $A_{i}(x, y)$ represents sub-aperture formed by $i$ th mirror satellite and $N$ means the number of mirror satellites. The center of the $A_{i}(x, y)$ is $\left(x_{c i}, y_{c i}\right)$ and the radius of the $i$ th sub-aperture is $r_{i}$. Therefore, in the synthetic aperture telescope system, the PSF can be affected by the formation of mirror satellites.

As described in section 2, the PSF of the synthetic aperture telescope system is changed by formation of mirror satellites. In the case of the formation is regular hexagon, the aperture, the PSF, and the modulation transfer function (MTF) are as shown in Figure 3, respectively. Where, monochromatic observation in wavelength $\lambda$ is assumed. The six circles on the upper left of the figure represent the position of the sub-aperture formed by the mirror satellites, and the circle of the circumscribed dotted line represents the aperture formed by the synthetic aperture. The PSF shown in the upper right of the figure shows multiple side lobes around the central peak due to the sparse aperture. The dotted circle in the figure shows the Airy disk of the single-mirror optical system of the same size aperture. In the case of an aberration free optical system using a single mirror, most of the energy collected is concentrated within this area. The figure on lower left shows the 2-dimensional distribution of the MTF. The MTF is calculated by the Fourier transform of the PSF, and orthogonal coordinate axes represent spatial frequencies. The dotted circle represents the optical cutoff frequency of an aberrationfree single-mirror optical system with the same diameter as the synthetic aperture. That is, in the case of an ideal monolithic mirror optical system, the information within the spatial frequency represented by the dotted line is included in observed images. On the other hand, in the case of the synthetic aperture telescope, as indicated by dark blue in the figure, there are many frequency regions where information cannot be obtained inside the dotted circle. Therefore, although the maximum observable spatial frequency of the synthetic aperture telescope is the same as that of the monolithic mirror telescope, there 
is information that is lost at lower spatial frequencies. The lower right figure shows a vertical section through the center of the MTF. The figure shows that the MTF response is lost over a wide range.

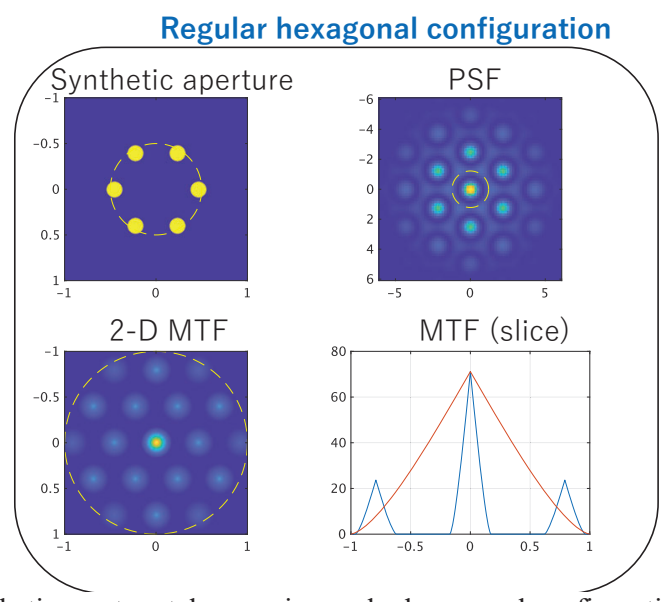

Figure 3. Optical properties of a synthetic aperture telescope in regular hexagonal configuration. (upper left) The position of mirror satellites. The dotted circle represents a single mirror with the same diameter as the synthetic aperture telescope. (upper right) The PSF of the synthetic aperture telescope. The dotted circle represents an Airy disk of the large aperture monolithic primary. (lower left) The 2-D MTF of the system. The dotted circle represents the optical cutoff frequency of the large aperture monolithic primary. (lower right) A vertical section through the center of the MTF normalized at the center. The red line shows the MTF of the large aperture monolithic primary.

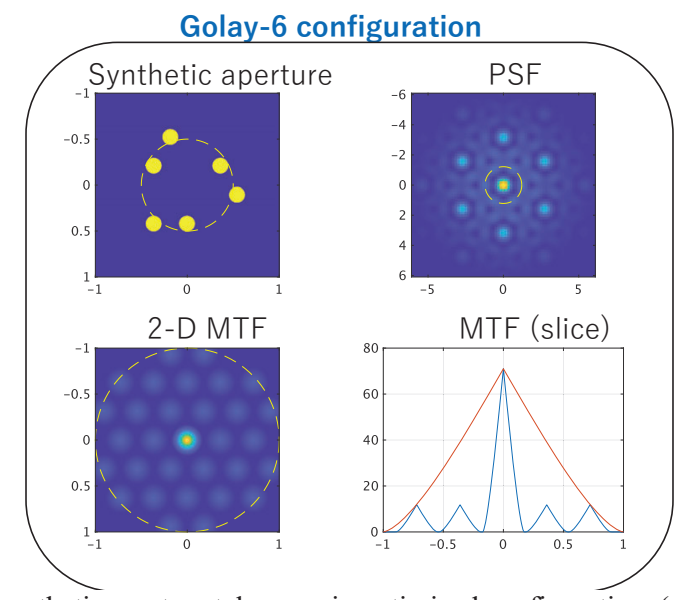

Figure 4. Optical performance of synthetic aperture telescope in optimized configuration. (upper left) The position of mirror satellites. The dotted circle represents a single mirror with the same diameter as the synthetic aperture telescope. (upper right) The PSF of the synthetic aperture telescope. The dotted circle represents an Airy disk of the large aperture monolithic primary. (lower left) The 2-D MTF of the system. The dotted circle represents the optical cutoff frequency of the large aperture monolithic primary. (lower right) A vertical section through the center of the MTF normalized at the center. The red line shows the MTF of the large aperture monolithic primary.

Next, we defined the evaluation function as the area of the region in spatial frequency domain, where the value of the MTF is below the threshold. The positions of the six mirror satellites were determined to minimize the value of this evaluation function, then the optimal mirror satellite formation was determined. The result was almost the same arrangement as Golay6 configuration. Here, all spatial frequencies were weighted equally, so the optimal arrangement was consistent with that 
of Golay-6. In the method used here, it is possible to determine the configuration of mirror satellites that increase the MTF value for the particular spatial frequency region by weighting specific spatial frequencies. Depending on the application, this technique can be useful when specific spatial frequency information is needed.

The calculation results for the optimal configuration are shown in Figure 4. Comparing the PSF shown in upper right of Figure 4 with the PSF shown in Figure 3, the value of peak of each side lobe is clearly reduced and the shape of the PSF is closer to that of a monolithic mirror telescope. According to the MTF in the lower left of Figure 4, it is not possible to eliminate the unresponsive area within the cutoff frequency, but it is understood that the area is smaller compared to the MTF in Figure 3.

Generally, objects are estimated by applying Wiener filter to the observed image, the distribution of the system PSF, and the noise characteristics of the sensor system, but the information of the object is lost in the region where the value of OTF is zero. As shown in this section, optimization of the mirror satellite arrangement minimizes the loss of information in the spatial frequency domain and improves the image restoration accuracy.

\section{USE OF OBSERVATION IMAGES WITH SYNTHETIC APERTURE TELESCOPE}

Figure 5 shows the numerical simulation results of the observed images based on the mathematical model of the formulated optical system. Assuming observations on the ground from geosynchronous orbit, numerical simulations were performed using the parameters in Table 1. The observed images were calculated by applying the PSFs of a large-diameter single mirror, a small-diameter single mirror, and a synthetic aperture telescope arranged in a Golay-6 configuration. We performed a numerical calculation of the observation of a point light source in close proximity, assuming forest fire detection. In this calculation, regions of high brightness of less than one pixel are distributed several pixels apart.

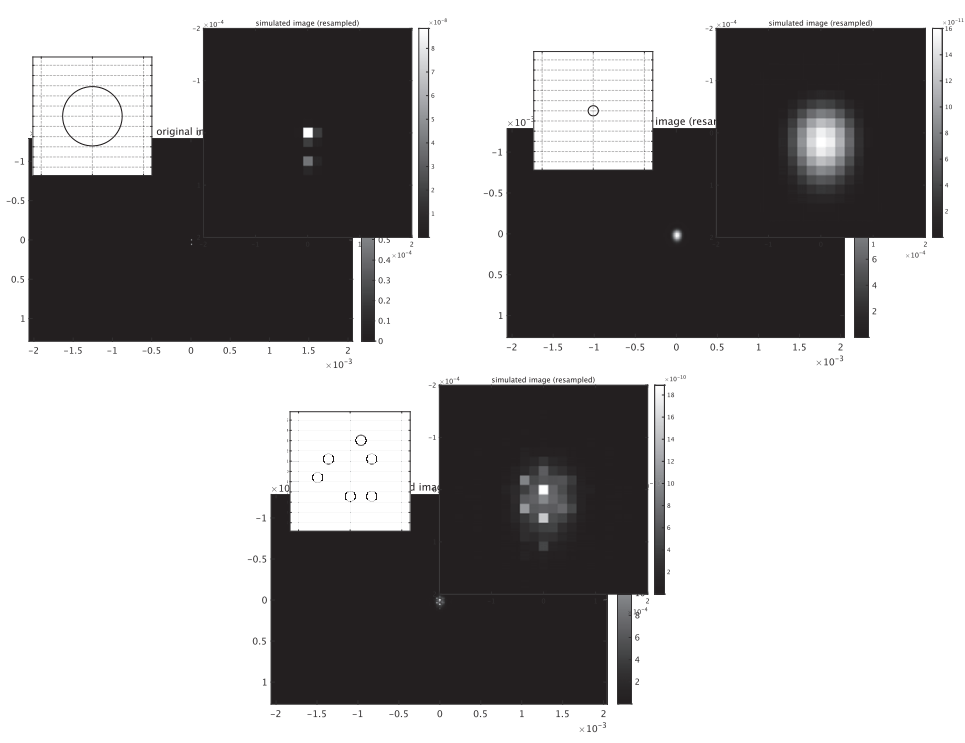

Figure 5. Numerical simulation results of images observing two aligned high-intensity point sources from GEO. (upper left) Images with a large diameter single mirror with the same diameter as the synthetic aperture system. (upper right) Images with a single mirror satellite. (lower) Images with a synthetic aperture system.

The upper left of Figure 5 shows an image of two high-intensity pixels representing the initial stages of a fire on the ground observed by a single mirror with a large aperture having the same radius as the synthetic aperture configuration. In this image, two pixels of high intensity can be completely distinguished. In an image of the same object observed by a single 
mirror satellite, the two pixels are indistinguishable and bright areas are distributed over a wide area. On the other hand, when the same objects are observed with synthetic apertures configuration, the two high-intensity pixels are clearly distinguishable, although some side lobes are observed. By using the a priori PSF distribution, it is possible to distinguish between the target and the side lobes as well.

Next, we simulated the observed image when we observed an urban area using same optical systems. An example of the calculation results is shown in Figure 6 In the image with the synthetic aperture telescope, multiple side lobes appear distributed over the range of the PSF for the small aperture. Consequently, at first glance, the images observed are similar to those obtained with a small-aperture telescope. However, when we focused on the details of the image, a fine structure could be observed in the image.
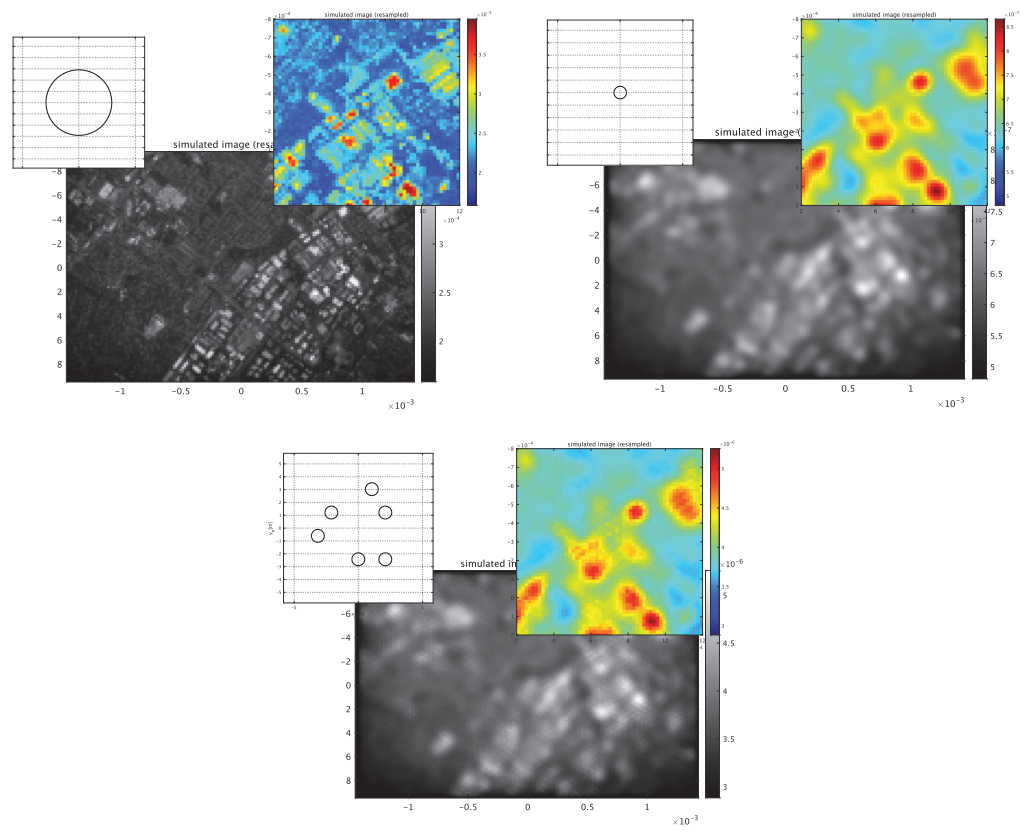

Figure 6. Numerical simulation results of images observing urban area from GEO. (upper left) Images with a large diameter single mirror with the same diameter as the synthetic aperture system. (upper right) Images with a single mirror satellite. (lower) Images with a synthetic aperture system.

We calculated the spatial spectrum of the observed image with the synthetic aperture configuration. The two-dimensional spectrum in the spatial frequency domain is shown in Figure 7. A high-pass filter was applied to the spectrum to cut off the spectral region obtained by the small-aperture single mirror, in order to extract the information obtained by the enlarged aperture using the synthetic aperture. The spatial spectrum was calculated by applying a window function. By enlarging the aperture using synthetic aperture, spectral information is obtained even outside the spatial frequency obtained by a single mirror. Appropriate extraction of information in this spatial frequency domain makes it possible to obtain resolutions not available with a single mirror satellite.

Figure 8 shows the result of reconstructing the image from the high spatial frequency spectrum and extracting the high spatial frequency information. The left image shows a part of the synthetic aperture image. In the middle image is the reconstructed image after applying a high-pass filter. In this image, the region with a value larger than the reference value was extracted as the region with high spatial frequency. The position of the region is indicated by the red $\mathrm{x}$ symbol in the figure. In addition, the extracted positions are superimposed on the image of the large aperture single mirror to show the detection results. Through a series of processing, a high spatial frequency region was extracted from an image that at first glance appears to be as blurry as that of the small aperture monocular. 


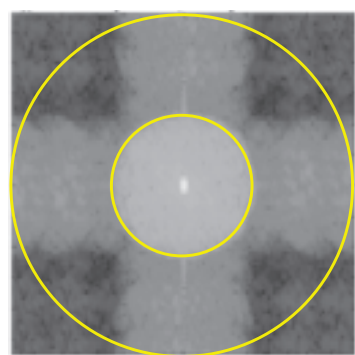

Figure 7. Spatial spectrum of the image obtained by synthetic aperture with high-pass filter. The inner circle represents the optical cutoff frequency of the aperture of a single mirror satellite, while the outer circle represents that of the synthetic aperture system.
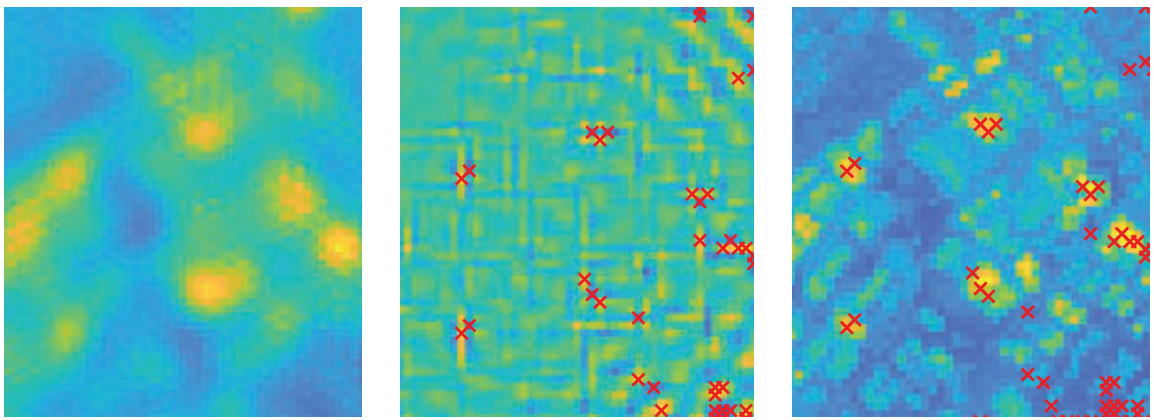

Figure 8. Information extraction result of synthetic aperture image, (left) synthetic start image, (middle) high spatial frequency domain extraction by high-pass filter, (right) superimposition of extraction position on high-resolution image.

\section{CONCLUSION}

We proposed the synthetic aperture telescope, FFSAT, which reconstructs a virtual large-aperture telescope by arranging small-aperture mirrors in an orbit using formation flying. As an optical feature of the synthetic aperture telescope, it is difficult to obtain general high-resolution images due to the sparseness of the primary mirror, but the images contain highspatial-resolution information. However, the images contain high spatial resolution information. We have shown a method to extract this information by image processing in the spatial frequency domain, and to identify the regions in the image where the high spatial resolution information appears. For example, in the case of wildfire detection, it is possible to extract the location of a wildfire with a high spatial frequency from a mountainous area with a low contrast. Our method will be more useful in identifying the location with high spatial frequency information than the precise image itself.

\section{REFERENCES}

[1] Bolcar, M. R., \& Fienup, J. R. (2009). Sub-aperture piston phase diversity for segmented and multi-aperture systems. Applied Optics, 48(1), A5-A12.

[2] Goodman, J. (2004). Introduction to Fourier Optics. Roberts \& Company Publishers.

[3] Mugnier, L., Cassaing, F., Sorrente, B., Baron, F., Velluet, M. T., Michau, V., \& Rousset, G. (2004). MULTIPLE APERTURE OPTICAL TELESCOPES: SOME KEY ISSUES FOR EARTH OBSERVATION FROM A GEO ORBIT. 5th International Conference On Space Optics, 15, 2. 